\title{
Correction to: Social support as a moderator of healthcare adherence and distress in long-term hematopoietic cell transplantation survivors
}

Kristina Holmegaard Nørskov ${ }^{1} \cdot$ Jean C. Yi $^{2} \cdot$ Marie-Laure Crouch $^{2} \cdot$ Allison Stover Fiscalini $^{3} \cdot$ Mary E. D. Flowers ${ }^{2,4} \cdot$ Karen L. Syrjala ${ }^{2,4}$

Published online: 7 April 2021

(C) Springer Science+Business Media, LLC, part of Springer Nature 2021

\section{Correction to: J Cancer Surviv} https://doi.org/10.1007/s11764-020-00979-4

The original version of the article has contained mistakes in Tables 2 and 4.

The authors regret that three negative signs are missing in Table 2 and in Table 4 two $p$-values are missing.

The corrected tables are given below.

The online version of the original article can be found at https://doi.org/ 10.1007/s11764-020-00979-4

Kristina Holmegaard Nørskov

Kristina.holmegaard.noerskov@ regionh.dk

1 Department of Hematology, Copenhagen University Hospital, Rigshospitalet, Blegdamsvej 9, 2100 Copenhagen Ø, Denmark

2 Clinical Research Division, Fred Hutchinson Cancer Research Center, Seattle, WA, USA

3 University of California at San Francisco, San Francisco, CA, USA

4 University of Washington School of Medicine, Seattle, WA, USA 
Table 2 Univariate relationship between hypothesized factors associated with distress and adherence

\begin{tabular}{|c|c|c|c|c|}
\hline \multirow[t]{2}{*}{ Variable } & \multicolumn{2}{|c|}{ Health care adherence } & \multicolumn{2}{|c|}{ Cancer and treatment distress } \\
\hline & Test value & $p$ value & Test value & $p$ value \\
\hline \multicolumn{5}{|l|}{ Covariates } \\
\hline Gender & $8.144^{\mathrm{a}}$ & $<0.001$ & $3.320 \mathrm{a}$ & 0.001 \\
\hline Age $(<40,40-64,>65)$ & $14.67^{\mathrm{b}}$ & $<0.001$ & $3.048^{\mathrm{b}}$ & 0.048 \\
\hline Years post-transplant $(<10, \geq 10)$ & $2.084^{\mathrm{a}}$ & 0.038 & $3.063^{\mathrm{a}}$ & 0.002 \\
\hline Income $\$(<40.000, \geq 40.000)$ & $4.266^{\mathrm{a}}$ & $<0.001$ & $2.676^{\mathrm{a}}$ & 0.008 \\
\hline $\begin{array}{l}\text { Education }(<4 \text { year college, } \\
\geq 4 \text { year college })\end{array}$ & $2.670^{\mathrm{a}}$ & 0.008 & $1.369^{\mathrm{a}}$ & 0.171 \\
\hline Marital status (married/partner, single/divorced, widowed) & $3.302^{\mathrm{a}}$ & $<0.001$ & $1.091^{\mathrm{a}}$ & 0.276 \\
\hline Donor source (autologous, allogeneic) & $3.304^{\mathrm{a}}$ & 0.001 & $1.658^{\mathrm{a}}$ & 0.098 \\
\hline Urbanไrural & $1.907^{\mathrm{a}}$ & 0.057 & $0.021^{\mathrm{a}}$ & 0.983 \\
\hline \multicolumn{5}{|l|}{ Health stressors } \\
\hline Comorbidity $(<3, \geq 3)$ & $3.695^{\mathrm{a}}$ & $<0.001$ & $4.735^{\mathrm{a}}$ & $<0.001$ \\
\hline cGVHD (moderate/severe, mild/none) & $2.683^{\mathrm{a}}$ & 0.009 & $5.196^{\mathrm{a}}$ & $<0.001$ \\
\hline \multicolumn{5}{|l|}{ Support } \\
\hline ESSI (continuous) & $0.134^{\mathrm{c}}$ & 0.001 & $-0.340^{\mathrm{c}}$ & $<0.001$ \\
\hline SAL (continuous) & $0.139^{\mathrm{c}}$ & $<0.001$ & $-0.310^{\mathrm{c}}$ & $<0.001$ \\
\hline \multicolumn{5}{|l|}{ Coping process } \\
\hline HSE (continuous) & $0.166^{\mathrm{c}}$ & $<0.001$ & $-0.404^{\mathrm{c}}$ & $<0.001$ \\
\hline
\end{tabular}

An association is measured using ${ }^{\mathrm{a}}$ independent $t$-test, ${ }^{\mathrm{b}}$ one-way ANOVA, ${ }^{\mathrm{c}}$ Pearson correlation. $c G V H D$ chronic graft versus host disease, ESSI ENRICHED Social Support Instrument, SAL social activity log, HSE health self-efficacy

Table 4 Multiple linear regression analysis predicting cancer and treatment distress

\begin{tabular}{|c|c|c|c|c|c|c|c|c|}
\hline & \multicolumn{2}{|c|}{ Block 1} & \multicolumn{2}{|c|}{ Block 2} & \multicolumn{2}{|c|}{ Block 3} & \multicolumn{2}{|c|}{ Block 4} \\
\hline & $\beta$ & $p$ value & $\beta$ & $p$ value & $\beta$ & $p$ value & $\beta$ & $p$ value \\
\hline \multicolumn{9}{|l|}{ Covariates } \\
\hline Gender $(\mathrm{ref}=\mathrm{male})$ & 0.12 & 0.002 & 0.12 & 0.001 & 0.13 & $<0.001$ & 0.13 & $<0.001$ \\
\hline Age & -0.004 & 0.924 & -0.05 & 0.202 & -0.05 & 0.12 & -0.009 & 0.794 \\
\hline Years post-transplant $(\geq 10$ years) & 0.09 & 0.023 & 0.06 & 0.116 & -0.06 & 0.108 & 0.07 & 0.047 \\
\hline Income $(\mathrm{ref}=\geq 40.000 \$)$ & 0.12 & 0.003 & 0.08 & 0.033 & 0.03 & 0.432 & -0.035 & 0.324 \\
\hline Donor source (ref=allogeneic) & 0.05 & 0.21 & 0.1 & 0.01 & 0.06 & 0.091 & 0.09 & 0.014 \\
\hline \multicolumn{9}{|l|}{ Health stressors } \\
\hline Comorbidity score $>3(\mathrm{ref}=<3)$ & & & 0.15 & $<0.001$ & 0.16 & $<0.001$ & 1.791 & $<0.001$ \\
\hline CGVHD moderate-severe $(\mathrm{ref}=$ none/mild) & & & 0.22 & $<0.001$ & 0.18 & $<0.001$ & 0.455 & 0.034 \\
\hline \multicolumn{9}{|l|}{ Social support moderators } \\
\hline ESSI (continuous)*Comorbidity score $>3(\mathrm{ref}=<3)$ & & & & & -0.567 & $<.001$ & -0.459 & $<0.001$ \\
\hline ESSI (continuous)*CCGVHD moderate-severe $(\mathrm{ref}=$ none/mild $)$ & & & & & 0.047 & 0.7 & 0.173 & 0.236 \\
\hline SAL (continuous) $*$ Comorbidity score $>3(\mathrm{ref}=<3)$ & & & & & -0.408 & $<0.001$ & -0.37 & $<0.001$ \\
\hline SAL (continuous)*CGVHD moderate-severe $(\mathrm{ref}=$ none/mild) & & & & & 0.033 & 0.7 & 0.07 & 0.41 \\
\hline \multicolumn{9}{|l|}{ Coping process moderators } \\
\hline HSE $($ continuous $) *$ Comorbidity score $>3(\mathrm{ref}=<3)$ & & & & & & & -0.883 & $<0.001$ \\
\hline HSE (continuous)*CCGVHD moderate-severe $(\mathrm{ref}=$ none/mild) & & & & & & & -0.511 & 0.006 \\
\hline $\mathrm{F}$ for block & 6.068 & $<0.001$ & 12.599 & $<0.001$ & 13.81 & $<0.001$ & 15.792 & $<0.001$ \\
\hline $\mathrm{R}^{2}$ for block & 0.04 & & 0.07 & & 0.06 & & 0.05 & \\
\hline F for full model & & & & & & & 36.18 & $<0.001$ \\
\hline $\mathrm{R}^{2} \Delta$ for full model & & & & & & & 0.22 & \\
\hline
\end{tabular}

$\beta$, standardized regression coefficient. $c G V H D$ chronic graft versus host disease, ESSI ENRICHED Social Support Instrument, SAL social activity log, HSE health self-efficacy

Publisher's note Springer Nature remains neutral with regard to jurisdictional claims in published maps and institutional affiliations. 